\title{
Do Correlates of Handgrip Strength Among the Elderly Ghanaian Population Matter? Empirical Evidence from WHO-SAGE Wave 1 Survey
}

\section{Sally Sonia Simmons}

Nacional'nyj issledovatel'skij universitet Vyssaa skola ekonomiki https://orcid.org/0000-0002-9126-5922

John Elvis Hagan Junior ( $\square$ elvis.hagan@uni-bielefeld.de )

https://orcid.org/0000-0003-3530-6133

\section{Bright Opoku Ahinkorah}

University of Technology Sydney

\section{Abdul-Aziz Seidu}

James Cook University

Thomas Schack

Universitat Bielefeld Fakultat fur Psychologie und Sportwissenschaft

\section{Research article}

Keywords: Handgrip, strength, elderly, population, Ghana

Posted Date: July 8th, 2020

DOI: https://doi.org/10.21203/rs.3.rs-40579/v1

License: (c) (i) This work is licensed under a Creative Commons Attribution 4.0 International License. Read Full License 


\section{Abstract}

Background: Recent empirical evidence suggests variations in handgrip strength in the elder years of life depending on multiple parameters, but such evidence is lacking in Ghana. The study determines what multiple factors are associated with handgrip strength among the older population in Ghana.

Method: Using data from the World Health organisation Global Ageing and Adult Health Survey (SAGE) Wave 1, four thousand, five hundred and ninety-six Ghanaians aged 50 and above years were selected through a multistage sampling technique in the country.

Results: It was revealed that handgrip strength decreased with age and on the average was lower among females. Older females had a weaker handgrip strength than their male counterparts. Handgrip strength was higher among persons who were not suffering from hypertension or arthritis. Additionally, weight (Model 1, [left hand $\{\nabla=0.95, p<0.01\}$, right hand

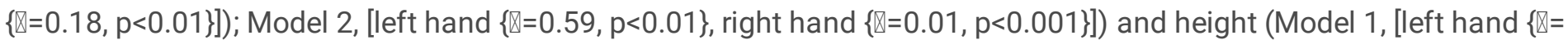

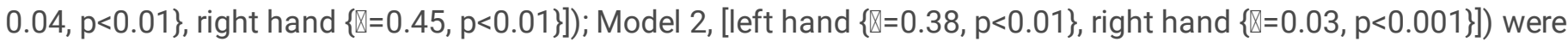
associated with increased grip strength.

Conclusions: Older adults' physical deterioration is inevitable because of structural and functional musculoskeletal limitations due to ageing. Hence, a timely identification of those at risk for appropriate intervention to promote their healthy living is necessary.

\section{Introduction}

Handgrip strength (HGS) is used as a benchmark for overall body muscle function and a proxy measure of physical health, particularly in older persons (1). It is a simple, non-invasive, reliable, and cost-effective screening technique for assessing muscle functioning (2). The measurement of HGS is also useful as a predictor of health status $(3,4)$, muscular strength, nutritional status, and disability (5). Besides, HGS has been used as a risk stratifying approach for causes of death and recovery time after illness or surgery (6).

Recent empirical evidence suggests variations in HGS of older people depending on multiple parameters such as sociodemographic factors (e.g., age, educational level, income, marital status, sex), anthropometric measurements (height, weight, body mass index (BMI) and muscle circumference), adverse health outcomes (e.g., depression, chronic diseases [e.g., diabetes, stroke and heart diseases]), and negative behavioural indices (e.g., physical inactivity, smoking, alcohol consumption, (7-9). For example, it has been established that men, generally, have higher HGS than women at all ages, with HGS higher in the right than the left hand in both sexes (10). Furthermore, HGS increases from childhood through adolescence, peaks at age $35 \pm 40$ years, and declines afterwards. Similarly, poor HGS is predictive of increased mortality from cardiovascular disease and cancer in men after controlling for muscle mass and body mass index (BMI) in some longitudinal studies $(11,12)$. There are also associations between HGS and chronic diseases as well as multi-morbidity in men and women, even when some confounding factors have been adjusted (13). Mostly, HGS is associated with disability in daily lives, walking speed and quality of life $(14,15)$.

Ageing is associated with muscle mass loss which can lead to a decrease in muscle strength (7). Decrease in strength because of ageing is mostly secondary to deterioration in skeletal muscle mass in men and women (16). Evidence suggests a direct relationship between decreasing muscle strength and age-related health complications, including degenerative diseases $(3,17,18)$. Furthermore, maintaining muscle strength, by regular involvement in physical activities, has been regarded as very essential in the reduction in functional limitations of older people (6). Other studies have identified an association between depression and muscle strength $(12,19,20)$.

Page $2 / 21$ 
Absolute estimates of the older population showed a sevenfold increase (i.e., from 215,258 in 1960 to 1,643,978 in 2010) in this population group in Ghana (21). Despite the ongoing ageing transition and evidence of the importance of HGS, public health professionals in the country focus more on maternal and child care (22). The health status of the aged can be assessed through a variety of indicators, including HGS, which is cost-effective and requires fewer efforts. Again, as an indicator, HGS supports early detection of diseases; malnutrition; functional disability and frailty, health challenges prevailing in the older years of life. However, to the best of our knowledge, information on HGS and its associated factors in Ghana is relatively lacking. The present study aimed to address this knowledge gap by examining the correlates of grip strength in the elderly population in Ghana. An empirical study on HGS and its associated factors is essential to enhance the health of the aged population and in the planning and implementation of public health initiatives and programs that aim to preserve the muscle strength of older people in the country.

\section{Methods}

Data

The data used for the study were obtained from the 2007-2008 World Health Organisation (WHO) Global Ageing and Adult Health, (SAGE) Wave 1 for Ghana. SAGE is a longitudinal study which collects data on adults aged 50 years and older, together with a comparable sample of adults aged 18-49 years, from nationally representative samples in six countries, Ghana inclusive. The survey was performed according to legal guidelines for carrying out population-based research in the country. SAGE Wave 1 recruited adults 18 years ad above using a multistage cluster sampling technique. The households became the clusters within which persons 18 years and above were selected. Together, a total of 5,110 respondents were involved in the survey. A detailed description of the SAGE survey, including data and its collection procedures, are provided on the project website.

\section{Variables}

The outcome variable for the study was handgrip strength for the right and left hands among the elderly, defined as the proportion of people aged 50 years and above who were either right-handed, left-handed or ambidextrous in Ghana. Eighteen variables were selected as predictor variables. These were sociodemographic (i.e., age, sex, marital status, and level of education), anthropometric (i.e., weight, height, hand dominance), economic (i.e., employment, wealth quintile), and health and behavioural variables (i.e., hypertension, diabetes, stroke, arthritis, fatigue, depression, physically active, tobacco use, and alcohol consumption). The selection of these variables was per previous studies and achievement of numerically stable and adaptable models (23). While age, height, weight, handgrip (left and right), were numeric variables, sex, employment, hypertension, diabetes, arthritis, tobacco use, alcohol consumption, and employment were dichotomous variables. Wealth quintile, hand dominance, fatigue, level of education and marital status were polychotomous.

Further, age was transformed into a categorical variable to reflect the significant stages of ageing and health (24). Marital status was assigned a different code with married as the initial status and never married as the final status. Respondents who were age 50 and above years and those who had a record of grip strength became the respondents for this study. Therefore the sample size of the study became 4596. Body mass index (BMI) was estimated using weight and height (in metres). As a measure of body fat, it was introduced to assess the influence of body-fat on health in the older years of life (25). Hence, this newly generated variable was added to those listed above. Missing values for the variables were imputed using the predictive mean matching multiple imputation approach. It helped to reduce the effects of missing data in all computations (26).

Data Analyses

Page $3 / 21$ 
The variables of interest were summarised to provide a clearer understanding of the distribution among males and females. The distribution was complemented with a test of variation in the outcome. Following this, a stepwise regression model was fitted to choose the best predictor variables from the variables- a backward elimination method was used for this purpose (27). The variables which generated the parsimonious model were included in the multivariate analysis. Linear regression (ordinary least squares-OLS) models were fitted for the analysis. Thus, multiple linear regression models were built to ascertain how each of the predictors contributed to the prediction of the mean value of the response variable (28). Three groups of linear models were fitted for the study. The first model used a standard linear regression. The second model included transformed variables in the form of logarithmic and interaction effects. The third model was a robust linear regression. Each of these models was repeated for the grip estimates of the left hand and right hand.

Model diagnostics were performed to explore problems that may compromise the regression analysis and determine whether certain assumptions appear reasonable (29). Autocorrelation was tested using the Durban-Watson test. ShapiroWilk test was used to assess the assumption of normality. The Breusch-Pagan test was used to assess the assumption of homogeneity of variance. Since the spread varied, the assumption of constant variance was violated. The presence of multicollinearity in the model was assessed using a variance inflation factor (VIF). The base for the VIF assessment was five and above. The model diagnostics' outcomes that did not appear reasonable were resolved using the fourth approach, fitting of robust models (30). These robust regressions were modelled for the standard linear regressions as well as the model with transformed variables. All analyses were weighted and performed using R programme. Statistical significance tests were based on a two-sided probability set at $p<0.05$.

\section{Results}

Descriptive statistics on socio-demographic and anthropometric characteristics

Data referent to all 4596 respondents is presented in Table 1. Generally, more males $(N=2308)$ than females $(N=2288)$ were included in the study. Majority of the respondents were aged 60-89 years (males $=50.4 \%$, females $=54.6 \%$ ) with a statistical difference between males and females. Many male respondents were married (83.0\%), whereas many females were widowed (46.5\%). While many males had secondary education (45.9\%), quite a proportion of females had no primary education (38.3\%). There were more right-handed respondents than others, although the right-hand female respondents (94.2\%) were more than males (92.8\%). Alcohol consumption and tobacco use were relatively lower among females (45.9\% and $7.7 \%$ ) than among males (69.5\% and $42.6 \%)$, but no statistical difference between males and females was recorded for alcohol consumption. Many females and males reported not having diabetes, even though more females (4.4\%) compared males (3.2\%) had diabetes. More females (64.7\%) than males (64.3\%) reported no fatigue. There was, however, no statistical difference between both sexes. There were differences in the distribution of wealth quintile for males and females, but no statistically significant difference was observed between males and females. 
Table 1

Sociodemographic characteristics of respondents by sex

\begin{tabular}{|c|c|c|c|c|c|c|c|c|}
\hline \multirow[t]{2}{*}{ Variables } & \multicolumn{2}{|c|}{ Males $(\mathrm{N}=2308)$} & & \multicolumn{3}{|c|}{ Females $(N=2288)$} & \multicolumn{2}{|c|}{ Total $(\mathrm{N}=4596)$} \\
\hline & $\mathrm{N}$ & $\%$ & $p$-value & $\mathrm{N}$ & $\%$ & $p$-value & $\mathrm{N}$ & $\%$ \\
\hline Age & & & $\star \star$ & & & $\star \star$ & & \\
\hline $50-59$ & 1116 & 48.4 & & 1006 & 44.0 & & 2122 & 46.2 \\
\hline $60-89$ & 1163 & 50.4 & & 1250 & 54.6 & & 2413 & 52.5 \\
\hline $90-115$ & 29 & 1.3 & & 32 & 1.4 & & 61 & 1.3 \\
\hline Marital Status & & & $\star \star \star$ & & & $\star \star \star$ & & \\
\hline Married & 1916 & 83.0 & & 763 & 33.3 & & 2679 & 58.3 \\
\hline Cohabiting & 21 & 0.9 & & 13 & 0.6 & & 34 & 0.7 \\
\hline Separated & 183 & 7.9 & & 417 & 18.2 & & 600 & 13.1 \\
\hline Widowed & 163 & 7.1 & & 1065 & 46.5 & & 1228 & 26.7 \\
\hline Never Married & 25 & 1.1 & & 30 & 1.3 & & 55 & 1.2 \\
\hline Level of Education & & & $\star \star \star$ & & & $\star \star \star *$ & & \\
\hline No education & 560 & 24.3 & & 877 & 38.3 & & 1437 & 31.3 \\
\hline Primary & 560 & 24.3 & & 652 & 28.5 & & 1212 & 26.4 \\
\hline Secondary & 1036 & 45.9 & & 681 & 29.8 & & 1717 & 37.3 \\
\hline Tertiary & 152 & 6.6 & & 78 & 3.4 & & 230 & 5.0 \\
\hline Hand Dominance & & & * & & & * & & \\
\hline Left & 140 & 6.1 & & 106 & 4.6 & & 246 & 5.4 \\
\hline Right & 2142 & 92.8 & & 2155 & 94.2 & & 4297 & 93.5 \\
\hline Both & 26 & 1.1 & & 27 & 1.2 & & 53 & 1.2 \\
\hline Tobacco use & & & $\star \star \star *$ & & & $\star \star \star *$ & & \\
\hline Yes & 984 & 42.6 & & 177 & 7.7 & & 1161 & 25.3 \\
\hline No & 1324 & 57.4 & & 2111 & 92.3 & & 3435 & 74.7 \\
\hline
\end{tabular}

Source: SAGE, Wave 1 (Ghana), 2007-2008

Note: $\mathrm{N}$ denotes number of observations

${ }^{*} \mathrm{p}<0.05,{ }^{*} \mathrm{p}<0.01,{ }^{* \star} \mathrm{p}<0.001 t$ test statistical significance between sex.

General and anthropometric data referent to all 4596 study participants is presented in Table 2. Mean weight was $68.20 \pm 46.08$, with no statistical difference between males and females. Mean height was $165.85 \pm 57.06$, with a statistically significant difference between males and females. The average grip strength for the left and right hands were $26.16 \pm 12.07$ and $27.46 \pm 12.01$, respectively. Weight $(69.97 \pm 46.40)$ and BMI $(24.29 \pm 12.95)$ were significantly greater in females whereas grip strength in both hands (left $=29.71 \pm 11.83$ and right $=31.05 \pm 11.78$ ) and height $(170.34 \pm 60.43)$ were greater in males. 


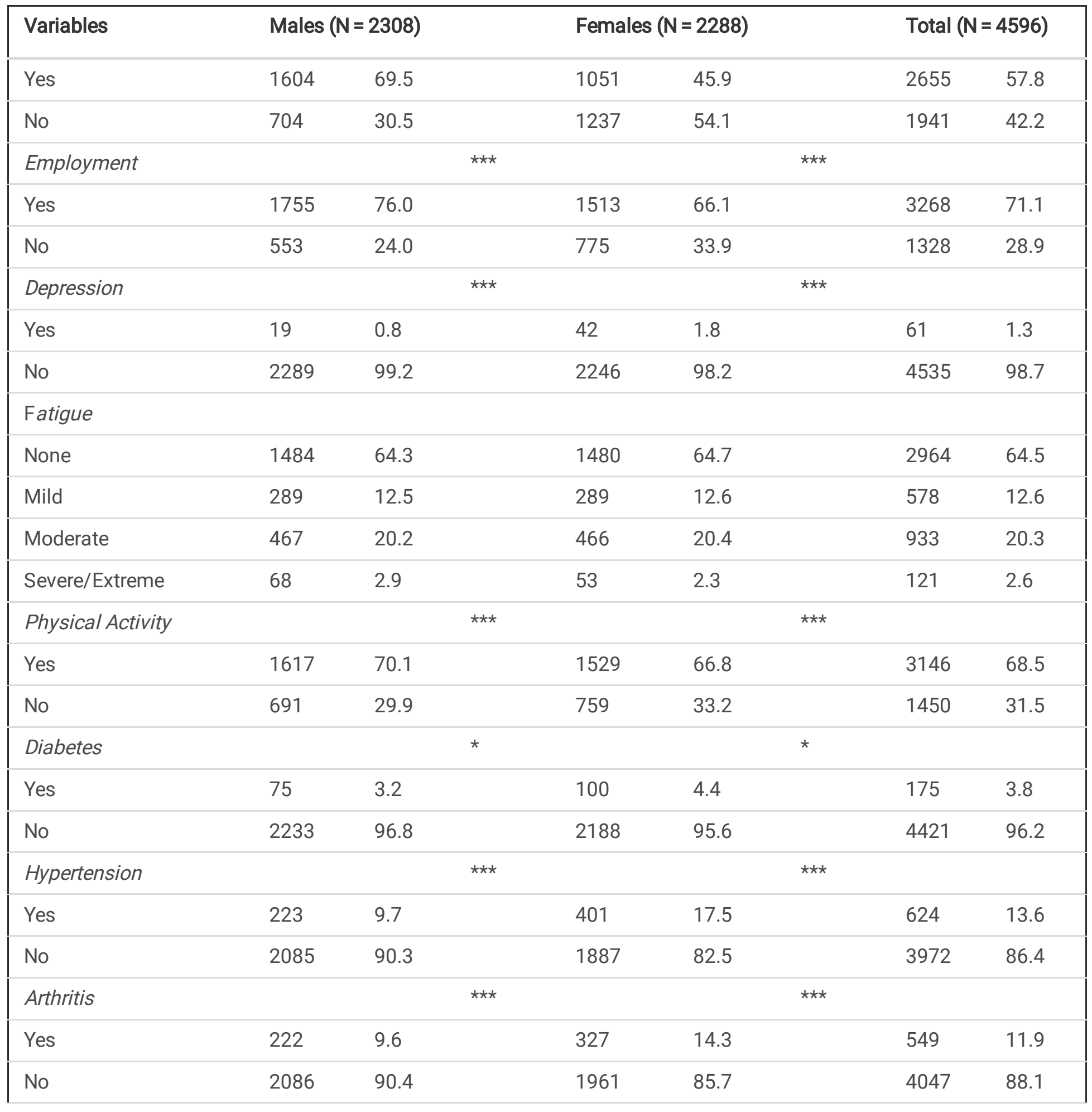

Source: SAGE, Wave 1 (Ghana), 2007-2008

Note: $\mathrm{N}$ denotes number of observations

${ }^{*} \mathrm{p}<0.05,{ }^{*} \mathrm{p}<0.01,{ }^{* \star} \mathrm{p}<0.001 t$ test statistical significance between sex.

General and anthropometric data referent to all 4596 study participants is presented in Table 2. Mean weight was $68.20 \pm 46.08$, with no statistical difference between males and females. Mean height was $165.85 \pm 57.06$, with a statistically significant difference between males and females. The average grip strength for the left and right hands were $26.16 \pm 12.07$ and $27.46 \pm 12.01$, respectively. Weight $(69.97 \pm 46.40)$ and BMI $(24.29 \pm 12.95)$ were significantly greater in females whereas grip strength in both hands (left $=29.71 \pm 11.83$ and right $=31.05 \pm 11.78$ ) and height $(170.34 \pm 60.43)$ were greater in males. 


\begin{tabular}{|c|c|c|c|c|c|c|}
\hline Variables & \multicolumn{2}{|c|}{ Males $(\mathrm{N}=2308)$} & \multicolumn{2}{|c|}{ Females $(\mathrm{N}=2288)$} & \multicolumn{2}{|c|}{ Total $(\mathrm{N}=4596)$} \\
\hline \multicolumn{7}{|l|}{ Stroke } \\
\hline Yes & 53 & 2.3 & 63 & 2.8 & 116 & 2.5 \\
\hline No & 2255 & 97.7 & 2225 & 97.2 & 4480 & 97.5 \\
\hline \multicolumn{3}{|l|}{ Wealth quintile } & \multicolumn{4}{|c|}{$\star \star \star *$} \\
\hline Lowest & 444 & 19.2 & 469 & 20.5 & 913 & 19.9 \\
\hline Lower & 421 & 18.2 & 479 & 20.9 & 900 & 19.6 \\
\hline Middle & 453 & 19.6 & 476 & 20.8 & 929 & 20.2 \\
\hline Higher & 466 & 20.2 & 453 & 19.8 & 919 & 20.0 \\
\hline Highest & 524 & 22.7 & 411 & 18.0 & 935 & 20.3 \\
\hline \multicolumn{7}{|c|}{ Source: SAGE, Wave 1 (Ghana), 2007-2008 } \\
\hline \multicolumn{7}{|c|}{ Note: $\mathrm{N}$ denotes number of observations } \\
\hline \multicolumn{7}{|c|}{${ }^{*} \mathrm{p}<0.05,{ }^{*} \mathrm{p}<0.01,{ }^{* \star *} \mathrm{p}<0.001$ t test statistical significance between sex. } \\
\hline \multicolumn{7}{|c|}{$\begin{array}{l}\text { General and anthropometric data referent to all } 4596 \text { study participants is presented in Table } 2 \text {. Mean weight was } \\
68.20 \pm 46.08 \text {, with no statistical difference between males and females. Mean height was } 165.85 \pm 57.06 \text {, with a } \\
\text { statistically significant difference between males and females. The average grip strength for the left and right hands } \\
\text { were } 26.16 \pm 12.07 \text { and } 27.46 \pm 12.01 \text {, respectively. Weight }(69.97 \pm 46.40) \text { and BMI }(24.29 \pm 12.95) \text { were significantly } \\
\text { greater in females whereas grip strength in both hands (left }=29.71 \pm 11.83 \text { and right }=31.05 \pm 11.78) \text { and height } \\
\text { (170.34 } \pm 60.43 \text { ) were greater in males. }\end{array}$} \\
\hline
\end{tabular}


Table 2

Anthropometric characteristics of respondents (by sex)

\begin{tabular}{|c|c|c|c|}
\hline Variables & Males $(\mathrm{N}=2308)$ & Females $(\mathrm{N}=2288)$ & Total $(\mathrm{N}=4596)$ \\
\hline & Mean \pm SD & Mean \pm SD & Mean \pm SD \\
\hline Weight & $66.42 \pm 45.74$ & $69.97 \pm 46.40$ & $68.20 \pm 46.08$ \\
\hline Height & $170.34 \pm 60.43^{\star \star \star}$ & $161.33 \pm 53.09 \star \star \star$ & $165.85 \pm 57.06$ \\
\hline BMI & 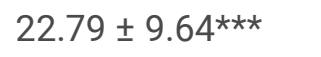 & $24.29 \pm 12.95^{\star \star \star}$ & $23.53 \pm 11.43$ \\
\hline Hand grip strength (left hand) & $29.71 \pm 11.83^{\star \star \star}$ & $22.59 \pm 11.21^{\star \star \star}$ & $26.16 \pm 12.07$ \\
\hline Hand grip strength (right hand) & $31.05 \pm 11.78^{\star \star \star}$ & $23.84 \pm 11.12^{\star \star \star}$ & $27.46 \pm 12.01$ \\
\hline \multicolumn{4}{|c|}{ Source: SAGE, Wave 1 (Ghana), 2007-2008 } \\
\hline \multicolumn{4}{|c|}{ Note: $\mathrm{N}$ denotes number of observations } \\
\hline \multicolumn{4}{|c|}{${ }^{*} \mathrm{p}<0.05,{ }^{* \star} \mathrm{p}<0.01,{ }^{* \star *} \mathrm{p}<0.001$ t test statistical significance between sex. } \\
\hline \multicolumn{4}{|c|}{ A Stepwise regression analysis on the association between predictor variables and grip strength } \\
\hline \multicolumn{4}{|c|}{ 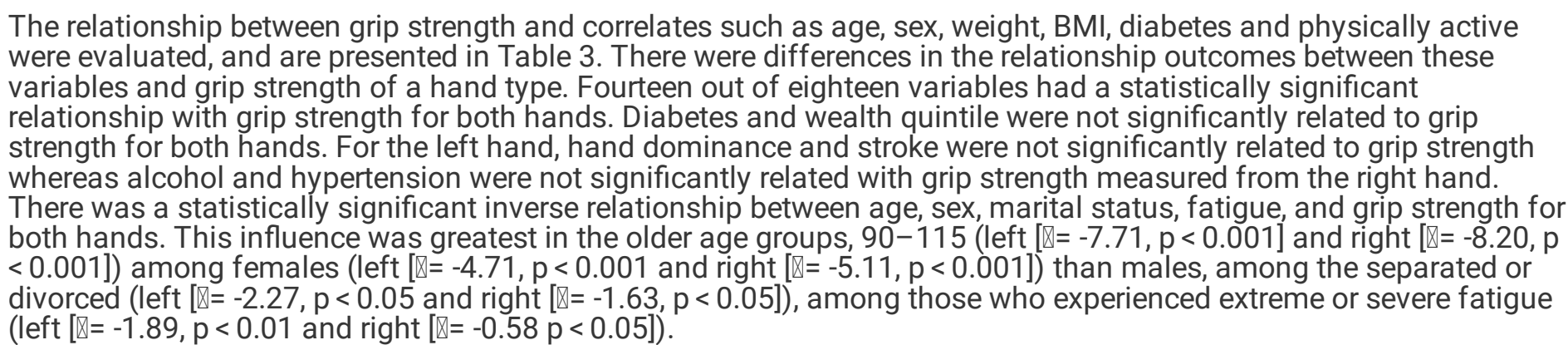 } \\
\hline
\end{tabular}


Table 3

Relationship between predictor variables and grip strength

\begin{tabular}{|c|c|c|c|c|}
\hline \multirow{2}{*}{ Variables } & \multicolumn{2}{|l|}{ Left hand } & \multicolumn{2}{|c|}{ Right hand } \\
\hline & $\otimes$ & $\mathrm{p}$ value & $\otimes$ & $\mathrm{p}$ value \\
\hline \multicolumn{5}{|l|}{ Age } \\
\hline $50-59$ & $a^{1}$ & $a^{1}$ & $a^{1}$ & $a^{1}$ \\
\hline $60-89$ & -2.90 & $<0.001$ & -3.34 & $<0.001$ \\
\hline $90-115$ & -7.71 & $<0.001$ & -8.20 & $<0.001$ \\
\hline \multicolumn{5}{|l|}{ Sex } \\
\hline Males & $a^{1}$ & $a^{1}$ & $a^{1}$ & $a^{1}$ \\
\hline Females & -4.71 & $<0.001$ & -5.11 & $<0.001$ \\
\hline \multicolumn{5}{|l|}{ Marital } \\
\hline Married & $a^{1}$ & $a^{1}$ & $a^{1}$ & $a^{1}$ \\
\hline Cohabiting & -1.13 & $<0.05$ & -0.65 & $<0.05$ \\
\hline Separated/Divorced & -2.27 & $<0.05$ & -1.63 & $<0.05$ \\
\hline Widowed & -1.28 & $<0.05$ & -1.44 & $<0.05$ \\
\hline Never married & -1.18 & $<0.05$ & -1.10 & $<0.05$ \\
\hline \multicolumn{5}{|l|}{ Education } \\
\hline No education & $a^{1}$ & $a^{1}$ & $a^{1}$ & $a^{1}$ \\
\hline Primary & 0.15 & $<0.05$ & 0.46 & $<0.05$ \\
\hline Secondary & 1.36 & $<0.05$ & 0.65 & $<0.05$ \\
\hline Tertiary & 1.81 & $<0.01$ & 0.72 & $<0.05$ \\
\hline \multicolumn{5}{|l|}{ Hand Dominance } \\
\hline Left & $a^{1}$ & $a^{1}$ & $a^{1}$ & $a^{1}$ \\
\hline Right & 0.21 & $<0.1$ & 2.99 & $<0.001$ \\
\hline Both & 1.83 & $<0.1$ & 4.050 & $<0.001$ \\
\hline \multicolumn{5}{|l|}{ Tobacco Use } \\
\hline Yes & $a^{1}$ & $a^{1}$ & $a^{1}$ & $a^{1}$ \\
\hline No & 0.73 & $<0.1$ & 0.72 & $<0.1$ \\
\hline \multicolumn{5}{|c|}{ Source: SAGE, Wave 1 (Ghana), 2007-2008 } \\
\hline \multicolumn{5}{|c|}{ Note: $\nabla$ denotes coefficients } \\
\hline \multicolumn{5}{|c|}{$a^{1}$ represents reference level } \\
\hline
\end{tabular}




\begin{tabular}{|c|c|c|c|c|}
\hline Variables & \multicolumn{2}{|l|}{ Left hand } & \multicolumn{2}{|c|}{ Right hand } \\
\hline \multicolumn{5}{|l|}{ Alcohol } \\
\hline Yes & $a^{1}$ & $a^{1}$ & $a^{1}$ & $a^{1}$ \\
\hline No & 0.19 & $<0.05$ & 0.37 & $<0.1$ \\
\hline \multicolumn{5}{|l|}{ Employment } \\
\hline Yes & $a^{1}$ & $a^{1}$ & $a^{1}$ & $a^{1}$ \\
\hline No & -1.25 & $<0.01$ & -0.52 & $<0.05$ \\
\hline \multicolumn{5}{|l|}{ Depression } \\
\hline Yes & $a^{1}$ & $a^{1}$ & $a^{1}$ & $a^{1}$ \\
\hline No & 7.30 & $<0.001$ & 7.31 & $<0.001$ \\
\hline \multicolumn{5}{|l|}{ Fatigue } \\
\hline None & $a^{1}$ & $a^{1}$ & $a^{1}$ & $a^{1}$ \\
\hline Mild & -1.10 & $<0.01$ & 0.41 & $<0.05$ \\
\hline Moderate & -1.12 & $<0.05$ & 0.47 & $<0.05$ \\
\hline Severe/Extreme & -1.89 & $<0.01$ & 0.58 & $<0.05$ \\
\hline \multicolumn{5}{|l|}{ Physically Active } \\
\hline Yes & $a^{1}$ & $a^{1}$ & $a^{1}$ & $a^{1}$ \\
\hline No & -0.84 & $<0.05$ & -1.43 & $<0.001$ \\
\hline \multicolumn{5}{|l|}{ Diabetes } \\
\hline Yes & $a^{1}$ & $a^{1}$ & $a^{1}$ & $a^{1}$ \\
\hline No & 0.31 & $<0.1$ & 0.38 & $<0.1$ \\
\hline \multicolumn{5}{|l|}{ Hypertension } \\
\hline Yes & $a^{1}$ & $a^{1}$ & $a^{1}$ & $a^{1}$ \\
\hline No & 1.38 & $<0.01$ & 0.66 & $<0.1$ \\
\hline \multicolumn{5}{|l|}{ Arthritis } \\
\hline Yes & $a^{1}$ & $a^{1}$ & $a^{1}$ & $a^{1}$ \\
\hline No & 5.72 & $<0.001$ & 5.97 & $<0.001$ \\
\hline \multicolumn{5}{|l|}{ Stroke } \\
\hline \multicolumn{5}{|c|}{ Source: SAGE, Wave 1 (Ghana), 2007-2008 } \\
\hline \multicolumn{5}{|c|}{ Note: $\nabla$ denotes coefficients } \\
\hline \multicolumn{5}{|c|}{$a^{1}$ represents reference level } \\
\hline$p<0.05, p<0.01$ & statistical & ificance & weer & ight hands \\
\hline
\end{tabular}




\begin{tabular}{|lllll|}
\hline Variables & \multicolumn{3}{l}{ Left hand } & \multicolumn{2}{l|}{ Right hand } \\
\hline Yes & $\mathrm{a}^{1}$ & $\mathrm{a}^{1}$ & $\mathrm{a}^{1}$ & $\mathrm{a}^{1}$ \\
\hline No & 0.92 & $<0.1$ & 2.18 & $<0.05$ \\
\hline Wealth quintile & & & $\mathrm{a}^{1}$ \\
\hline Lowest & $\mathrm{a}^{1}$ & $\mathrm{a}^{1}$ & $\mathrm{a}^{1}$ & $<0.1$ \\
\hline Lower & 0.18 & $<0.1$ & 0.85 & $<0.1$ \\
\hline Middle & 0.58 & $<0.1$ & 0.42 & $<0.1$ \\
\hline Higher & 0.61 & $<0.1$ & 0.34 & $<0.1$ \\
\hline Highest & 0.74 & $<0.1$ & 1.11 & $<0.05$ \\
\hline Weight & 0.02 & $<0.05$ & 0.01 & $<0.05$ \\
\hline Height & 0.01 & $<0.05$ & 0.04 & $<0.05$ \\
\hline BMI & 0.05 & $<0.05$ & 0.11 & \\
\hline Source: SAGE, Wave 1 (Ghana), 2007-2008 & & & \\
\hline Note: $\otimes$ denotes coefficients & & & \\
\hline $\mathrm{a}^{1}$ represents reference level & & & \\
\hline p $0.05, \mathrm{p}<0.01, \mathrm{p}<0.001$, statistical significance between left and right hands & \\
\hline
\end{tabular}

\section{Multiple linear regression analyses on the predictors of grip strength}

Table 4 depicts results on the predictors of grip strength among the elderly population in Ghana. The outcomes of the three groups of models are illustrated in the table. The first, second and third group of models are standard linear (Model 1), transformed linear (Model 2) and robust linear regression models (Model 3, A and B), respectively. From the models, all variables had statistically significant effects on grip strength. However, most of the significant predictors had an oscillating relationship with grip strength depending on the type of hand measured. Except for education, all demographic variables had an inverse association with grip strength. Grip strength was decreased with age, 60-89

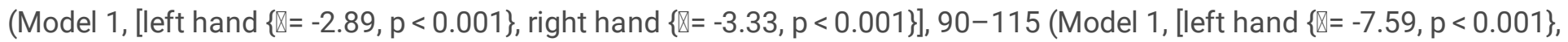

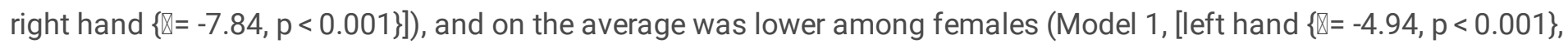
right hand $\{\mathbb{X}=-5.30, p<0.001\}])$. Grip strength was higher among persons who were not suffering from hypertension

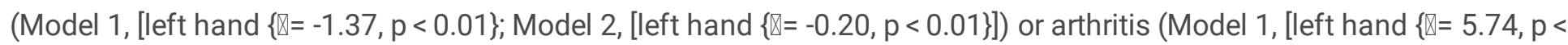

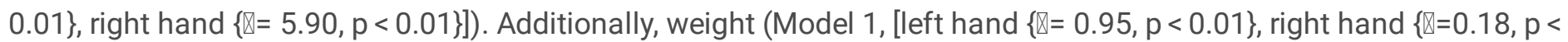

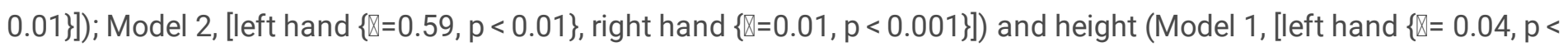

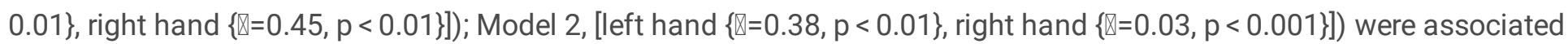
with increased grip strength. Older females had a weaker grip than males. The standard linear models had the least variation ( $R^{2}=45 \%$, each hand) in grip strength explained by the predictors. Model 2 showed a higher level of

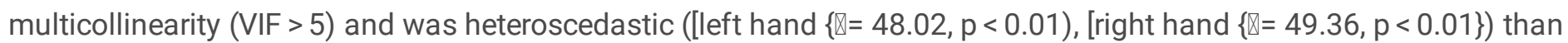

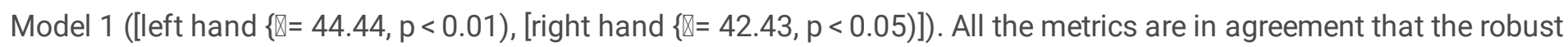
linear models with transformations (Model 3B) provide the best fit to the data (AIC [left hand]: 10447.64, AIC [right hand]: 10055.45). 
Table 4

Predictors of grip strength

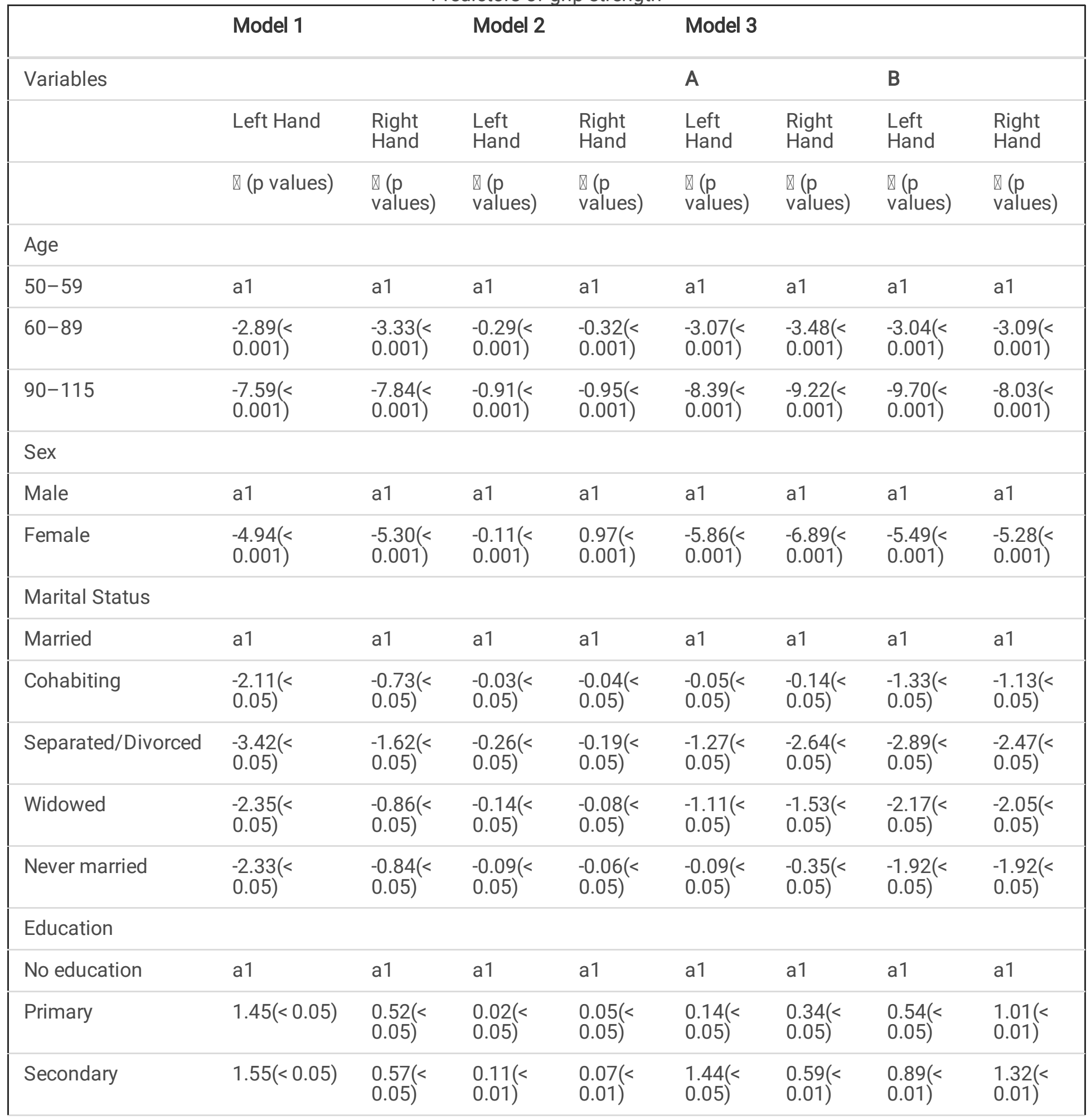

Source: SAGE, Wave 1 (Ghana), 2007-2008

Note: $\nabla$ denotes coefficients which appear before the $p$-values (indicated in the parentheses);

BMI represents body mass index;

Hand Dom represents hand dominance

log. represents logarithms 


\begin{tabular}{|c|c|c|c|c|c|c|c|c|}
\hline & Model 1 & & Model 2 & & Model 3 & & & \\
\hline Tertiary & $2.72(<0.01)$ & $\begin{array}{l}2.43(< \\
0.01)\end{array}$ & $\begin{array}{l}0.16(< \\
0.01)\end{array}$ & $\begin{array}{l}0.09(< \\
0.01)\end{array}$ & $\begin{array}{l}2.97(< \\
0.001)\end{array}$ & $\begin{array}{l}1.20(< \\
0.001)\end{array}$ & $\begin{array}{l}1.34(< \\
0.01)\end{array}$ & $\begin{array}{l}2.87(< \\
0.01)\end{array}$ \\
\hline \multicolumn{9}{|l|}{ Employment } \\
\hline Yes & a1 & a1 & a1 & a1 & a1 & a1 & a1 & a1 \\
\hline No & $\begin{array}{l}-1.19(< \\
0.01)\end{array}$ & $\begin{array}{l}-0.63(< \\
0.05)\end{array}$ & $\begin{array}{l}-0.09(< \\
0.05)\end{array}$ & $\begin{array}{l}-0.03(< \\
0.05)\end{array}$ & $\begin{array}{l}-1.46(< \\
0.001)\end{array}$ & $\begin{array}{l}-2.80(< \\
0.05)\end{array}$ & $\begin{array}{l}-1.22(< \\
0.05)\end{array}$ & $\begin{array}{l}-1.31(< \\
0.001)\end{array}$ \\
\hline \multicolumn{9}{|l|}{ Depression } \\
\hline Yes & a1 & a1 & a1 & a1 & a1 & a1 & a1 & a1 \\
\hline No & $\begin{array}{l}7.30(< \\
0.001)\end{array}$ & $\begin{array}{l}7.38(< \\
0.001)\end{array}$ & $\begin{array}{l}1.94(< \\
0.001)\end{array}$ & $\begin{array}{l}1.83(< \\
0.001)\end{array}$ & $\begin{array}{l}6.12(< \\
0.001)\end{array}$ & $\begin{array}{l}5.62(< \\
0.001)\end{array}$ & $\begin{array}{l}1.22(< \\
0.001)\end{array}$ & $\begin{array}{l}1.19(< \\
0.001)\end{array}$ \\
\hline \multicolumn{9}{|l|}{ Fatigue } \\
\hline None & a1 & a1 & a1 & a1 & a1 & a1 & a1 & a1 \\
\hline Mild & $\begin{array}{l}-1.26(< \\
0.01)\end{array}$ & $\begin{array}{l}-0.34(< \\
0.01)\end{array}$ & $\begin{array}{l}-0.10(< \\
0.01)\end{array}$ & $\begin{array}{l}-0.04(< \\
0.01)\end{array}$ & $\begin{array}{l}-5.80(< \\
0.05)\end{array}$ & $\begin{array}{l}-1.23(< \\
0.05)\end{array}$ & $\begin{array}{l}-1.08(< \\
0.01)\end{array}$ & $\begin{array}{l}-1.53(< \\
0.05)\end{array}$ \\
\hline Moderate & $\begin{array}{l}-1.77(< \\
0.01)\end{array}$ & $\begin{array}{l}-0.35(< \\
0.01)\end{array}$ & $\begin{array}{l}-0.12(< \\
0.01)\end{array}$ & $\begin{array}{l}-0.05(< \\
0.01)\end{array}$ & $\begin{array}{l}-8.01(< \\
0.05)\end{array}$ & $\begin{array}{l}-1.35(< \\
0.05)\end{array}$ & $\begin{array}{l}-1.11(< \\
0.01)\end{array}$ & $\begin{array}{l}-2.43(< \\
0.01)\end{array}$ \\
\hline Severe/Extreme & $\begin{array}{l}-1.89(< \\
0.01)\end{array}$ & $\begin{array}{l}-0.39(< \\
0.01)\end{array}$ & $\begin{array}{l}-0.14(< \\
0.01)\end{array}$ & $\begin{array}{l}-0.08(< \\
0.01)\end{array}$ & $\begin{array}{l}-9.61(< \\
0.05)\end{array}$ & $\begin{array}{l}-7.29(< \\
0.05)\end{array}$ & $\begin{array}{l}-2.48(< \\
0.01)\end{array}$ & $\begin{array}{l}-5.38(< \\
0.01)\end{array}$ \\
\hline \multicolumn{9}{|l|}{ Physically Active } \\
\hline Yes & a1 & a1 & a1 & a1 & a1 & a1 & a1 & a1 \\
\hline No & $-0.85(0.016)$ & $\begin{array}{l}-1.47(< \\
0.001)\end{array}$ & $\begin{array}{l}-0.47(< \\
0.001)\end{array}$ & $\begin{array}{l}-0.51(< \\
0.001)\end{array}$ & $\begin{array}{l}0.20(< \\
0.001)\end{array}$ & $\begin{array}{l}1.01(< \\
0.01)\end{array}$ & $\begin{array}{l}2.34(< \\
0.01)\end{array}$ & $\begin{array}{l}3.36(< \\
0.001)\end{array}$ \\
\hline \multicolumn{9}{|l|}{ Arthritis } \\
\hline Yes & a1 & a1 & a1 & a1 & a1 & a1 & a1 & a1 \\
\hline No & $\begin{array}{l}5.74(< \\
0.001)\end{array}$ & $\begin{array}{l}5.90(< \\
0.001)\end{array}$ & $\begin{array}{l}1.04(< \\
0.001)\end{array}$ & $\begin{array}{l}1.00(< \\
0.001)\end{array}$ & $\begin{array}{l}5.60(< \\
0.001)\end{array}$ & $\begin{array}{l}5.80(< \\
0.001)\end{array}$ & $\begin{array}{l}2.33(< \\
0.001)\end{array}$ & $\begin{array}{l}8.59(< \\
0.001)\end{array}$ \\
\hline Weight & $0.95(<0.05)$ & $\begin{array}{l}0.18(< \\
0.01)\end{array}$ & $\begin{array}{l}0.59(< \\
0.01)\end{array}$ & $\begin{array}{l}0.01(< \\
0.001)\end{array}$ & $\begin{array}{l}7.60(< \\
0.001)\end{array}$ & $\begin{array}{l}8.96(< \\
0.001)\end{array}$ & $\begin{array}{l}4.72(< \\
0.001)\end{array}$ & $\begin{array}{l}5.82(< \\
0.001)\end{array}$ \\
\hline Height & $0.04(<0.05)$ & $\begin{array}{l}0.45(< \\
0.05)\end{array}$ & $\begin{array}{l}0.38(< \\
0.01)\end{array}$ & $\begin{array}{l}0.03(< \\
0.001)\end{array}$ & $\begin{array}{l}4.25(< \\
0.001)\end{array}$ & $\begin{array}{l}4.49(< \\
0.001)\end{array}$ & $\begin{array}{l}2.43(< \\
0.001)\end{array}$ & $\begin{array}{l}3.59(< \\
0.001)\end{array}$ \\
\hline BMI & $0.89(<0.05)$ & $\begin{array}{l}0.11(< \\
0.05)\end{array}$ & $\begin{array}{l}0.02(< \\
0.05)\end{array}$ & $\begin{array}{l}0.01(< \\
0.05)\end{array}$ & $\begin{array}{l}1.81(< \\
0.05)\end{array}$ & $\begin{array}{l}1.59(< \\
0.05)\end{array}$ & $\begin{array}{l}2.54(< \\
0.05)\end{array}$ & $\begin{array}{l}2.72(< \\
0.05)\end{array}$ \\
\hline \multicolumn{9}{|l|}{ Stroke } \\
\hline \multicolumn{9}{|c|}{ Source: SAGE, Wave 1 (Ghana), 2007-2008 } \\
\hline \multicolumn{9}{|c|}{ Note: $\otimes$ denotes coefficients which appear before the p-values (indicated in the parentheses); } \\
\hline \multicolumn{9}{|c|}{ BMI represents body mass index; } \\
\hline \multicolumn{9}{|c|}{ Hand Dom represents hand dominance } \\
\hline log. represents & & & & & & & & \\
\hline
\end{tabular}


Model 1

Model 2

a1

$2.10(<$

$0.05)$ a1

$0.41(<$

$0.001)$

\section{Model 3}

a1

a1

$4.90(<$

$4.88(<$

$0.001)$

Alcohol

\begin{tabular}{|c|c|c|c|c|}
\hline Yes & a1 & a1 & a1 & a1 \\
\hline No & $0.05(<0.05)$ & $\begin{array}{l}0.02(< \\
0.05)\end{array}$ & $\begin{array}{l}0.54(< \\
0.01)\end{array}$ & $\begin{array}{l}0.67(< \\
0.05)\end{array}$ \\
\hline
\end{tabular}

Hypertension

$\begin{array}{lllll}\text { Yes } & \text { a1 } & \text { a1 } & \text { a1 } & \text { a1 } \\ \text { No } & 1.37(<0.01) & 0.20(< & 1.15(< & 1.08(< \\ & & 0.001) & 0.001) & 0.05)\end{array}$

Hand Dom.

$\begin{array}{lllll}\text { Left } & \text { a1 } & \text { a1 } & \text { a1 } & \text { a1 } \\ \text { Right } & 2.99(< & 0.29(< & 3.25(< & 3.84(< \\ & 0.001) & 0.001) & 0.001) & 0.001) \\ \text { Both } & 5.95(< & 5.93(< & 4.93(< & 4.19(< \\ & 0.001) & 0.01) & 0.01) & 0.01)\end{array}$

Transformations

Log. Weight

$3.01(<\quad 2.80(<$

6.57 (<

7.45(<

0.001) 0.001)

0.001) 0.001)

Log. Height

$\begin{array}{ll}0.53(< & 0.74(< \\ 0.001) & 0.001)\end{array}$

3.09 (<

$2.90(<$

0.001) 0.001$)$

Phy. Act. \& Arth

No Phy.: Act: No

$-0.43(<\quad-0.44(<$

0.001) $\quad 0.001)$

$-2.64(<$

$-2.98(<$

0.01) 0.01)

Sex \& Arthritis

Female: Arthritis

$\begin{array}{ll}-0.24(< & -0.21(< \\ 0.05) & 0.05)\end{array}$

$-2.92(<$

$-2.56(<$

$0.01)$

0.01)

Age \& Sex

60-89: Female

$-0.06(<\quad-0.06(<$

$-0.72(<$

$0.01)$

$-0.32(<$

0.01 )

$0.05)$

$-0.54(<\quad-0.58(<$

-1.49 (< $\quad-0.48(<$

90-115:Female

0.01 )

0.05)

0.01 )

$0.001)$

Source: SAGE, Wave 1 (Ghana), 2007-2008

Note: $\nabla$ denotes coefficients which appear before the $p$-values (indicated in the parentheses);

BMI represents body mass index;

Hand Dom represents hand dominance

log. represents logarithms 


\begin{tabular}{|c|c|c|c|c|c|c|c|c|}
\hline & \multicolumn{2}{|l|}{ Model 1} & \multicolumn{2}{|l|}{ Model 2} & \multicolumn{4}{|l|}{ Model 3} \\
\hline \multicolumn{9}{|l|}{ Sex \& Depression } \\
\hline Female: No & & & $\begin{array}{l}-1.42(< \\
0.001)\end{array}$ & $\begin{array}{l}-1.30(< \\
0.001)\end{array}$ & & & $\begin{array}{l}-2.84(< \\
0.01)\end{array}$ & $\begin{array}{l}-2.84(< \\
0.001)\end{array}$ \\
\hline \multicolumn{9}{|l|}{ Diagnostics } \\
\hline Heteroscedasticity & $\begin{array}{l}44.44(< \\
0.01)\end{array}$ & $\begin{array}{l}42.43(< \\
0.05)\end{array}$ & $\begin{array}{l}48.02(< \\
0.05)\end{array}$ & $\begin{array}{l}49.36(< \\
0.05)\end{array}$ & & & & \\
\hline Autocorrelation & $\begin{array}{l}1.65(< \\
0.001)\end{array}$ & $\begin{array}{l}1.69(< \\
0.001)\end{array}$ & $\begin{array}{l}1.65(< \\
0.001)\end{array}$ & $\begin{array}{l}1.69(< \\
0.001)\end{array}$ & & & & \\
\hline Normality & $\begin{array}{l}0.93(< \\
0.001)\end{array}$ & $\begin{array}{l}0.94(< \\
0.001)\end{array}$ & $\begin{array}{l}0.93(< \\
0.001)\end{array}$ & $\begin{array}{l}0.94(< \\
0.001)\end{array}$ & & & & \\
\hline VIF & $<5$ & $<5$ & $>5$ & $>5$ & & & & \\
\hline Intercept & $\begin{array}{l}11.42(< \\
0.001)\end{array}$ & $\begin{array}{l}13.68(< \\
0.001)\end{array}$ & $\begin{array}{l}-13.14(< \\
0.001)\end{array}$ & $\begin{array}{l}-13.23(< \\
0.001)\end{array}$ & $\begin{array}{l}12.35(< \\
0.001)\end{array}$ & $\begin{array}{l}13.13(< \\
0.001)\end{array}$ & $\begin{array}{l}-15.44(< \\
0.001)\end{array}$ & $\begin{array}{l}-16.21(< \\
0.001)\end{array}$ \\
\hline F-value & $\begin{array}{l}64.30(< \\
0.001)\end{array}$ & $\begin{array}{l}62.54(< \\
0.001)\end{array}$ & $\begin{array}{l}64.98(< \\
0.001)\end{array}$ & $\begin{array}{l}65.33(< \\
0.001)\end{array}$ & & & & \\
\hline $\mathrm{R}^{2}$ & 0.45 & 0.45 & 0.58 & 0.59 & 0.62 & 0.65 & 0.67 & 0.69 \\
\hline \multicolumn{9}{|c|}{ Source: SAGE, Wave 1 (Ghana), 2007-2008 } \\
\hline \multicolumn{9}{|c|}{ Note: $\nabla$ denotes coefficients which appear before the p-values (indicated in the parentheses); } \\
\hline \multicolumn{9}{|c|}{ BMI represents body mass index; } \\
\hline \multicolumn{9}{|c|}{ Hand Dom represents hand dominance } \\
\hline log. represents loga & & & & & & & & \\
\hline
\end{tabular}

Phy. Act. represents physically active

Arth. represents arthritis

VIF represents multicollinearity

$a^{1}$ represents reference level

$p<0.05, p<0.01, p<0.001$, statistical significance between left and right hands

\section{Discussion}

Handgrip strength (HGS) is a simple and inexpensive marker of skeletal muscle function among older adults. The purpose of this study was to examine the correlates of grip strength in the elderly population in Ghana based on data from the 2007-2008 World Health Organisation (WHO) Global Ageing and Adult Health, (SAGE) Wave 1 for Ghana. Males' left hand $(29.71 \pm 11.83)$ and right hand $(31.05 \pm 11.78)$ HGS mean values were higher than females' left-hand $(22.59 \pm 11.21)$ and right hand $(23.84 \pm 11.12)$ HGS mean values respectively. Mean weight $(69.97 \pm 46.40)$ and $\mathrm{BMI}$ $(24.29 \pm 12.95)$ were significantly higher in females, whereas mean height (170.34 \pm 60.43$)$ was higher in males than females' (161.33 \pm 53.09$)$. Findings also show an age-dependent decline in HGS for both genders across handedness (i.e., left and right hand) from the 6th) decade (i.e., 60+) and beyond, but the pattern of decline in HGS with advancing 
age was more pronounced (i.e., lower) among females. HGS was higher among persons who were not hypertensive and suffered arthritis. Among the three anthropometric measures, increased HGS was significantly associated with weight and height. These findings are indications that HGS is influenced by multifaceted factors which were confirmed through the regression analyses.

The aged have their bodies' manifest age-related degenerative variations in the musculoskeletal, vascular, and nervous systems regardless of gender. These degenerative changes usually cause the decline of hand function in older adults and subsequently influence their hand structure such as joints, muscle, tendon, bone, nerve and receptors, blood supply, skin, and fingernails (31). Consistent with previous studies, the decrease of muscle strength across handedness because of ageing cannot be ruled out regarding the HGS, and ageing association noted in the current study. Two primary reasons account for HGS loss during ageing. First, there is a decrease in muscle mass because of functional loss of motor units (32), resulting in an incomplete re-innervation of abandoned muscle fibres by surviving motor neurons (33) and selective atrophy of fast-twitch muscle fibres in the hand (34). The second is the reduced capacity to adequately stimulate muscles in the hand during ageing (32). Other mechanisms include joint stiffness, reduction in muscle coordination, decreasing physical activity, decreasing hormone levels, and chronic diseases that come with advancing age (35-37). However, sex variations in HGS mechanisms underlying ageing are not well understood (38). According to Kim et al. (9); Lenardt et al. (38), HGS in women degenerates intensely from $55 \pm 59$ years onwards. These authors note that sex hormones are vital in explaining this sex-related variance. It has been proven that muscle strength is similar in men and premenopausal women, although there is a sudden deterioration in muscle strength from menopause thereafter (39). Additionally, sex differences in HGS may be explained by hereditary sex variations in muscle mass $(40,41)$.

The finding that HGS was higher among persons free from hypertension or arthritis suggests that low grip strength is associated with increased susceptibility in persons who might develop cardiovascular disease (42) and some degenerative diseases (e.g., osteoarthritis). Loss of muscular strength might likely be part of the causal link to cardiovascular and other degenerative diseases. The current study did not, however, directly predict the inverse association between HGS and incident hypertension or arthritis and it possible that unmeasured parameters like nutritional status (43), endothelial dysfunction and autonomic imbalance $(44,45)$, as well as arterial stiffness, might mediate the relationship between HGS and cardiovascular events $(42,46)$. Future studies are required to provide a clearer understanding of whether increased HGS directly reduces the risk of incident cardiovascular disease and other degenerative diseases.

Similar to other research, significant associations were identified between increased HGS with height and weight in older adults in the current study (47-49). Regarding height, Stulp et al. (50) showed that taller people have absolute superior strength compared to shorter individuals. Absolute strength is associated with cross-sectional muscle area. It is related to one's body surface area or the square of an individual's body height and a factor that is more closely linked to lean mass (i.e., muscles, bone and non-fat tissues), a characteristic associated with muscle strength (51). According to Chandrasekaran et al. (52), persons with greater heights have longer arms, with greater arm leverage for force generation, resulting in an adequate amount of force. For weight, older people who have low body weight have low muscle mass and hence weaker physical strength, a biomarker of poor HGS. Lower muscle mass could also be associated with undernutrition or chronic disuse often connected to older age (6).

\section{Practical implications}

Ageing has a degenerative impact on hand function, including HGS. Therefore, HGS is a marker of the frailty phenotype in older adults (37). By entering the sample values of the predictor variables (i.e., age, sex, height, weight, BMI) into the regression models, specific estimates of Ghanaian older adults' left and right grip strength were provided. These estimates might be useful for clinicians or researchers who use normative values within their working software to provide 
more accurate predictions of normative strength scores for specific applications in different populations (53). The study findings suggest that assessment of HGS in elderly Ghanaians who are free from cardiovascular illness (e.g., hypertension) and degenerative disease (e.g., arthritis) could also be a risk biomarker for the incidence of these diseases across the Ghanaian aged population and perhaps other similar geographical locations. Future studies through longitudinal designs are required to examine how older people with low HGS or muscle strength may be assisted to improve their structural and functional musculoskeletal representation. To promote healthy ageing, helping elderly Ghanaian people to maintain physical function at old age is vital. On the bases of current findings, interventions to improve the HGS through regular physical activity (e.g., Activities of Daily Living, [ADL]), appropriate nutritional intake and other health care programmes for the aged are required (54). Sub-group level physical activity and elderly care programmes could prioritize women who were identified as the most affected with lower HGS compared to their male counterparts.

\section{Strengths and limitations}

To best of our knowledge, this is the first population-based study to describe the correlates and pattern of HGS loss across age and gender in a large representative cohort of Ghanaian people. The large sample size recruited from the general population and the use of rigorous analysis through the regressions models for the measurement variables give generalizability and credibility to the results as well as findings. These findings could be used as baseline data for context-specific interventions and more research. However, the cross-sectional age profiles of HGS across the sample may either under or overestimate individual-specific declines.

Further research is required through longitudinal designs using multiple momentary ecological assessments to give more accurate predictions of the magnitude of grip strength loss with age. There are other factors (e.g., arm length, grip force, grip speed, grip endurance, hand dominance, muscle mass) that are connected to HGS and were not investigated in the current study. Therefore, current findings should be noted with caution.

\section{Conclusions}

HGS is a vital marker of future health-compromising outcomes (e.g., reduced physical function- gait-related problems [impaired mobility], cardiovascular disease [hypertension]). It is very essential for the maintenance of healthy ageing and independence as well as a significant prerequisite for proper hand function. The present study demonstrated that sociodemographic (i.e., age, sex) and anthropometric (i.e., height, weight) correlate with HGS among the elderly population in Ghana. Older adults' physical deterioration is inevitable because of structural and functional musculoskeletal limitations due to ageing. Hence, a timely identification of those at risk for appropriate intervention to promote their healthy living is necessary. The reported mean values for HGS across age and gender with different height and weight could offer clinicians with baseline information when assessing the elderly. These reference values would also provide useful information for the general public to observe the HGS function of this vulnerable target group for early assistance.

\section{Abbreviations}

BMI

Body Mass Index

HGS

Handgrip Strength

SAGE

World Health Organisation (WHO) Global Ageing and Adult Health Survey

ADL

Activities of Daily Living

Page $17 / 21$ 


\section{Declarations}

\section{Ethics approval and consent to participate}

Not applicable

\section{Conflicts of Interests}

The authors declare that there are no competing interests.

\section{Funding}

There was no funding for the study.

\section{Authors' contributions}

SSS conceived the study. SSS, JEH, BOA, TS and AAS drafted the manuscript. All authors read and approved the final the manuscript.

\section{Acknowledgments}

Not Applicable

\section{Data and Material Availability}

The data used for the present study can be accessed from the project website

\section{References}

1. Clegg A, Young J, Iliffe S, Rikkert MO, Rockwood K. Frailty in elderly people. Lancet. 2013 Mar;2(9868):752-62. 381(.

2. Bohannon RW, Schaubert KL. Test-retest reliability of grip-strength measures obtained over a 12-week interval from community-dwelling elders. J Hand Ther. 2005 Dec;18(4):426-7. quiz 428.

3. Bohannon RW. Hand-grip dynamometry predicts future outcomes in aging adults. J Geriatr Phys Ther. 2008;31(1):310.

4. Heimbürger O, Qureshi AR, Blaner WS, Berglund L, Stenvinkel P. Hand-grip muscle strength, lean body mass, and plasma proteins as markers of nutritional status in patients with chronic renal failure close to start of dialysis therapy. Am J Kidney Dis. 2000 Dec;36(6):1213-25.

5. Bohannon RW. Muscle strength: clinical and prognostic value of hand-grip dynamometry. Curr Opin Clin Nutr Metab Care. 2015 Sep;18(5):465-70.

6. Moy F, Chang E, Kee K. Predictors of Handgrip Strength among the Free Living Elderly in Rural Pahang, Malaysia. Iran J Public Health. 2011 Dec 31;40(4):44-53.

7. Damayanthi HDWT, Moy F-M, Abdullah KL, Dharmaratne SD. Handgrip Strength and Its Associated Factors among Community-dwelling Elderly in Sri Lanka: A Cross-sectional Study. Asian Nursing Research. 2018 Sep 1;12(3):231-6.

8. Fukumori N, Yamamoto Y, Takegami M, Yamazaki S, Onishi Y, Sekiguchi M, et al. Association between hand-grip strength and depressive symptoms: Locomotive Syndrome and Health Outcomes in Aizu Cohort Study (LOHAS). Age Ageing. 2015 Jul;44(4):592-8.

9. Lenardt MH, Binotto MA, Carneiro NHK, Cechinel C, Betiolli SE, Lourenço TM, et al. Handgrip strength and physical activity in frail elderly. Revista da Escola de Enfermagem da USP. 2016 Feb;50(1):86-92. 
10. Wong SL. Grip strength reference values for Canadians aged 6 to 79: Canadian Health Measures Survey, 2007 to 2013. Health Rep. 2016 Oct;19(10):3-10. 27(.

11. Little MA. Grip strength and body composition in Turkana pastoralist children and adolescents. American Journal of Human Biology. 2017;29(2):e22922.

12. Rantanen T, Volpato S, Ferrucci L, Heikkinen E, Fried LP, Guralnik JM. Handgrip strength and cause-specific and total mortality in older disabled women: exploring the mechanism. J Am Geriatr Soc. 2003 May;51(5):636-41.

13. Cheung C-L, Nguyen U-SDT, Au E, Tan KCB, Kung AWC. Association of handgrip strength with chronic diseases and multimorbidity: a cross-sectional study. Age (Dordr). 2013 Jun;35(3):929-41.

14. Chan OYA, van Houwelingen AH, Gussekloo J, Blom JW, den Elzen WPJ. Comparison of quadriceps strength and handgrip strength in their association with health outcomes in older adults in primary care. Age (Dordr). 2014;36(5):9714.

15. Wagner PR, Ascenço S, Wibelinger LM, Wagner PR, Ascenço S, Wibelinger LM. Hand grip strength in the elderly with upper limbs pain. Revista Dor. 2014 Sep;15(3):182-5.

16. Bassi R, Sharma S, Kaur S, Sharma A. Handgrip dynamometry in elderly individuals and its relation with body mass index. National Journal of Physiology Pharmacy Pharmacology. 2016;6(6):599-603.

17. McPhee JS, French DP, Jackson D, Nazroo J, Pendleton N, Degens H. Physical activity in older age: perspectives for healthy ageing and frailty. Biogerontology. 2016 Jun;17(3):567-80.

18. Seene T, Kaasik P. Muscle weakness in the elderly: role of sarcopenia, dynapenia, and possibilities for rehabilitation. Eur Rev Aging Phys Act. 2012 Oct 1;9(2):109-17.

19. Visser M, Deeg DJH, Lips P, Longitudinal Aging Study Amsterdam. Low vitamin D and high parathyroid hormone levels as determinants of loss of muscle strength and muscle mass (sarcopenia): the Longitudinal Aging Study Amsterdam. J Clin Endocrinol Metab. 2003 Dec;88(12):5766-72.

20. Lauretani F, Semba RD, Bandinelli S, Dayhoff-Brannigan M, Giacomini V, Corsi AM, et al. Low Plasma Carotenoids and Skeletal Muscle Strength Decline over Six Years. J Gerontol A Biol Sci Med Sci. 2008 Apr;63(4):376-83.

21. 10.1057/978-1-137-60243-5

Huq M, Tribe M. The Economy of Ghana: 50 Years of Economic Development [Internet]. London: Palgrave Macmillan UK; 2018 [cited 2020 Jun 27]. Available from: http://link.springer.com/10.1057/978-1-137-60243-5.

22. Zere E, Kirigia JM, Duale S, Akazili J. Inequities in maternal and child health outcomes and interventions in Ghana. BMC Public Health. 2012 Mar 31;12(1):252.

23. Fraiman R, Justel A, Svarc M. Selection of Variables for Cluster Analysis and Classification Rules. J Am Stat Assoc. 2008 Sep;103(483):1294-303.

24. Sigelman CK, Rider EA. Life-Span Human Development. Cengage Learning; 2014. 770 p.

25. Nuttall FQ. Body Mass Index. Nutr Today. 2015;50(3):117-28.

26. Sterne JAC, White IR, Carlin JB, Spratt M, Royston P, Kenward MG, et al. Multiple imputation for missing data in epidemiological and clinical research: potential and pitfalls. BMJ. 2009 Sep;338(jun29 1)(1):b2393-3.

27. Ing C-K, Lai TL. A Stepwise Regression Method and Consistent Model Selection For High-Dimensional Sparse Linear Models. Statistica Sinica. 2011;21(4):1473-513.

28. Chao Y-CE, Zhao Y, Kupper LL, Nylander-French LA. Quantifying the Relative Importance of Predictors in Multiple Linear Regression Analyses for Public Health Studies. Journal of Occupational and Environmental Hygiene. 2008 Jul;5(8):519-29.

29. Altman N, Krzywinski M. Regression diagnostics. Nature Methods. 2016 May 1;13(5):385-6.

30. Casson RJ, Farmer LD. Understanding and checking the assumptions of linear regression: a primer for medical researchers: Assumptions of linear regression. Clinical Experimental Ophthalmology. 2014 Aug;42(6):590-6. 
31. Carmeli E, Patish H, Coleman R. The Aging Hand. J Gerontol A Biol Sci Med Sci. 2003 Feb 1;58(2):M146-52.

32. Siparsky PN, Kirkendall DT, Garrett WE. Muscle Changes in Aging. Sports Health. 2014 Jan;6(1):36-40.

33. Unguez GA, Bodine-Fowler S, Roy RR, Pierotti DJ, Edgerton VR. Evidence of incomplete neural control of motor unit properties in cat tibialis anterior after self-reinnervation. J Physiol (Lond). 1993 Dec;472:103-25.

34. Wang Y, Pessin JE. Mechanisms for fiber-type specificity of skeletal muscle atrophy. Curr Opin Clin Nutr Metab Care. 2013 May;16(3):243-50.

35. Larsson L, Degens H, Li M, Salviati L, Lee Y il, Thompson W, et al. Sarcopenia: Aging-Related Loss of Muscle Mass and Function. Physiol Rev. 2019 Jan 1;99(1):427-511.

36. Al Snih S, Markides KS, Ray L, Ostir GV, Goodwin JS. Handgrip strength and mortality in older Mexican Americans. J Am Geriatr Soc. 2002 Jul;50(7):1250-6.

37. Syddall H, Cooper C, Martin F, Briggs R, Aihie Sayer A. Is grip strength a useful single marker of frailty? Age Ageing. 2003 Nov;32(6):650-6.

38. Kim YH, So W-Y. Anthropometrics and Metabolic Syndrome in Healthy Korean Adults: A 7-Year Longitudinal Study. J Mens Health. 2018;14(4):E1-10.

39. Phillips SK, Rook KM, Siddle NC, Bruce SA, Woledge RC. Muscle weakness in women occurs at an earlier age than in men, but strength is preserved by hormone replacement therapy. Clin Sci. 1993 Jan;84(1):95-8.

40. Zaccagni L, Toselli S, Bramanti B, Gualdi-Russo E, Mongillo J, Rinaldo N. Handgrip Strength in Young Adults: Association with Anthropometric Variables and Laterality. IJERPH. 2020 Jun 15;17(12):4273.

41. Gallup AC, O'Brien DT, White DD, Wilson DS. Handgrip Strength and Socially Dominant Behavior in Male Adolescents. Evol Psychol. 2010 Apr 1;8(2):147470491000800200.

42. Leong DP, Teo KK, Rangarajan S, Kutty VR, Lanas F, Hui C, et al. Reference ranges of handgrip strength from 125,462 healthy adults in 21 countries: a prospective urban rural epidemiologic (PURE) study. J Cachexia Sarcopenia Muscle. 2016 Dec;7(5):535-46.

43. Norman K, Stobäus N, Gonzalez MC, Schulzke J-D, Pirlich M. Hand grip strength: outcome predictor and marker of nutritional status. Clin Nutr. 2011 Apr;30(2):135-42.

44. Taylor AC, McCartney N, Kamath MV, Wiley RL. Isometric training lowers resting blood pressure and modulates autonomic control. Med Sci Sports Exerc. 2003 Feb;35(2):251-6.

45. Clarkson P, Montgomery HE, Mullen MJ, Donald AE, Powe AJ, Bull T, et al. Exercise training enhances endothelial function in young men. J Am Coll Cardiol. 1999 Apr;33(5):1379-85.

46. Yamamoto K, Kawano H, Gando Y, lemitsu M, Murakami H, Sanada K, et al. Poor trunk flexibility is associated with arterial stiffening. American Journal of Physiology-Heart and Circulatory Physiology. 2009 Aug 7;297(4):H1314-8.

47. Keevil VL, Luben R, Dalzell N, Hayat S, Sayer AA, Wareham NJ, et al. Cross-sectional associations between different measures of obesity and muscle strength in men and women in a British cohort study. J Nutr Health Aging. 2015 Jan 1;19(1):3-11.

48. Ramlagan S, Peltzer K, Phaswana-Mafuya N. Hand grip strength and associated factors in non-institutionalised men and women 50 years and older in South Africa. BMC Res Notes. 2014 Jan 7;7:8.

49. Shim JH, Roh SY, Kim JS, Lee DC, Ki SH, Yang JW, et al. Normative measurements of grip and pinch strengths of 21st century korean population. Arch Plast Surg. 2013 Jan;40(1):52-6.

50. Stulp G, Buunk AP, Verhulst S, Pollet TV. Human Height Is Positively Related to Interpersonal Dominance in Dyadic Interactions. PLoS One [Internet]. 2015 Feb 26 [cited 2020 Jun 27];10(2). Available from:

https://www.ncbi.nlm.nih.gov/pmc/articles/PMC4342156/.

51. Luna-Heredia E, Martín-Peña G, Ruiz-Galiana J. Handgrip dynamometry in healthy adults. Clin Nutr. 2005 Apr;24(2):250-8.

Page 20/21 
52. Chandrasekaran B, Ghosh A, Prasad C, Krishnan K, Chandrasharma B. Age and anthropometric traits predict handgrip strength in healthy normals. J Hand Microsurg. 2010 Dec;2(2):58-61.

53. Lim SH, Kim YH, Lee JS. Normative Data on Grip Strength in a Population-Based Study with Adjusting Confounding Factors: Sixth Korea National Health and Nutrition Examination Survey (2014-2015). IJERPH. 2019 Jun 25;16(12):2235.

54. Arokiasamy P, Selvamani Y. Age, socioeconomic patterns and regional variations in grip strength among older adults (50+) in India: Evidence from WHO's Study on Global Ageing and Adult Health (SAGE). Arch Gerontol Geriatr. 2018 Jun;76:100-5. 